\title{
Ureterocele ectópica em cão: relato de caso
}

[Ectopic ureterocele in a dog: a case report]

\author{
C.A.B. Lorigados ${ }^{1}$, A.M. Zoppa ${ }^{1}$, L.A.G.P. Silva ${ }^{2}$, V. De Marco ${ }^{3}$, F. Futema ${ }^{2}$, \\ R.M.T. Silva ${ }^{4}$, L.O. Queiroz ${ }^{4}$ \\ ${ }^{1}$ Faculdade de Medicina Veterinária - UniFMU- Unicsul, SP \\ ${ }^{2}$ Faculdade de Medicina Veterinária - UnG,SP \\ ${ }^{3}$ Faculdade de Medicina Veterinária - UNISA, SP \\ ${ }^{4}$ Médica veterinária residente - Hovet - UnG, SP
}

\begin{abstract}
RESUMO
Relataram-se o quadro clínico, o diagnóstico, o tratamento e o acompanhamento de um cão com ureterocele ectópica e hidronefrose/hidroureter associados. Após as informações obtidas nos exames laboratoriais e de diagnóstico por imagem, o animal foi submetido à ureterocelectomia e à neoureterostomia. O cão apresentou evolução favorável após a conduta terapêutica. Apesar de pouco frequente, a ureterocele deve ser considerada como diagnóstico diferencial em animais jovens com histórico de incontinência urinária.
\end{abstract}

Palavras-chave: cão, ureterocele, ureteres, incontinência urinária

\begin{abstract}
The clinical features, diagnosis, treatment and monitoring of a dog with an ectopic ureterocele and concomitant hydronephrosis/hydroureter were reported. After the information obtained in laboratory tests and imaging diagnosis, the animal was submitted to ureterocelectomy and neoureterostomy. The dog presented a favorable evolution after the therapy. Although infrequent, the ureterocele should be considered a differential diagnosis in young animals with history of urinary incontinence.
\end{abstract}

Keywords: dog, ureterocele, ureters, urinary incontinence

\section{INTRODUÇÃO}

Normalmente os ureteres inserem-se de forma oblíqua na região do trígono vesical e seguem em um curto trajeto entre a camada muscular e a mucosa da vesícula urinária, antes de abrirem o orifício ureteral no interior do lúmen vesical (Dyce et al., 2010). A ureterocele é uma afecção congênita, que se caracteriza pela dilatação cística do segmento terminal do ureter, geralmente localizada em seu trajeto intramural e que se protrui para o lúmen vesical (McLoughlin et al., 1989; Lautzenhiser e Bjorling, 2002; Stiffler et al., 2002; Sutherland-Smith et al., 2004a; Green et al., 2011). Algumas classificações diferentes têm sido propostas para a ureterocele. A mais comumente utilizada classifica-a como ortotópica (ou intravesical), quando a dilatação cística e a abertura do orifício ureteral se localizam no interior da vesícula urinária, e ectópica, quando o orifício ureteral encontra-se em outra localização, como o colo vesical ou a uretra (McLoughlinet al., 1989; Lamb, 1998; Lautzenhiser e Bjorling, 2002). Stiffler et al. (2002) propuseram uma classificação levando em consideração não somente a alteração anatômica do ureter, mas também o grau de comprometimento funcional do rim (ausência ou presença de hidronefrose/hidroureter associados).

É uma doença pouco frequente no cão e mais rara ainda no gato (Eisele et al., 2005). As fêmeas são mais acometidas que os machos

Recebido em 31 de outubro de 2011

Aceito em 24 de maio de 2012

E-mail: clorigados@usp.br 
(Green et al., 2011), mas nenhuma predisposição racial tem sido relatada, sendo descrita em Poodle, Cocker Spaniel, Husky Siberiano, Scottish Terrier, Labrador e em cães sem raça definida (McLoughlin et al., 1989; Takiguchi et al., 1997; Lautzenhiser e Bjorling, 2002; Stiffler et al., 2002; Sutherland-Smith et al., 2004a; Colopy et al., 2011; Green et al., 2011; Secrest et al., 2011).

No homem, é comum as ureteroceles estarem associadas à duplicação do sistema coletor, ou seja, os rins apresentam duas pelves independentes, cada uma drenada por um ureter (Jaiman e Ulhoj, 2010). Nos pacientes veterinários portadores de ureterocele relatados até $\mathrm{o}$ momento, essa alteração não foi encontrada, e o acometimento, na maioria dos casos, é unilateral (Green et al., 2011).

A alteração clínica mais comumente associada à ureterocele é a incontinência urinária, embora a retenção urinária, a disúria e a polaquiúria também possam ser observadas (McLoughlin et al., 1989; Takiguchi et al., 1997; Lautzenhiser e Bjorling, 2002; Stiffler et al., 2002; SutherlandSutherland-Smith et al., 2004a; Adams e Syme, 2010; Green et al., 2011). Várias modalidades de imagem têm sido utilizadas no diagnóstico da ureterocele, como a ultrassonografia, a urografia excretora, a cistoscopia e a tomografia computadorizada (Takiguchi et al., 1997; Lamb, 1998; Siverman e Long, 2000; Sutherland-Smith et al., 2004a; Larsson, 2009;Green et al., 2011; Secrest et al., 2011). O tratamento da ureterocele é cirúrgico, e diferentes técnicas têm sido descritas, como a ureteronefrectomia, a neoureterostomia, a ureterocelectomia e a incisão por cistoscopia (Sutherland-Smith et al., 2004b; Tattersall e Welsh, 2006). O presente trabalho tem como objetivo relatar o quadro clínico, o diagnóstico, o tratamento e o acompanhamento de um cão com ureterocele ectópica.

\section{RELATO DE CASO}

Uma cadela, Poodle, de um ano de idade, foi atendida com histórico de incontinência urinária desde filhote. Não se observaram outras alterações significativas ao exame físico. Foram solicitados hemograma, função renal e urina tipo I. Os exames laboratoriais revelaram discreta leucocitose por neutrofilia, ureia e creatinina dentro dos padrões de normalidade e uma importante infecção urinária.

O animal foi submetido à ultrassonografia abdominal, na qual se observou uma formação cística preenchida por conteúdo anecoico, com parede fina e ecogênica no lúmen vesical ureterocele - (Fig. 1A). Foi possível identificar a dilatação do ureter esquerdo - $1 \mathrm{~cm}$ em segmento cranial e $0,51 \mathrm{~cm}$ em porção mais caudal - e sua inserção na região do trígono vesical, comunicando-se coma dilatação cística intravesical (Fig. 1B). A pelve do rim esquerdo encontrava-se bastante dilatada - hidronefrose severa -, com perda da arquitetura renal. Não foram observadas alterações sonográficas em rim e ureter direitos. $\mathrm{O}$ animal recebeu uma sonda para realização de cistografia com contraste iodado, após o exame ultrassonográfico. Nesse exame, foi possível observar a formação cística intravesical preenchida por contraste, prolongando-se para o colo vesical e a uretra. Houve refluxo do contraste para o ureter esquerdo (Fig. 2). Após o resultado de função renal, realizou-se a urografia excretora. Nesse exame, além da dilatação da pelve e do ureter esquerdos, foi possível observar que o rim esquerdo apresentava filtração, demonstrada pela presença do nefrograma e pielograma, embora o tempo de excreção do contraste estivesse alterado. A ureterocele se mostrou com uma falha de preenchimento de limites bem definidos em região caudal do lúmen vesical (Fig. 3). Não se detectaram alterações do rim direito, e o ureter correspondente apresentava diâmetro normal e inserção tópica. 


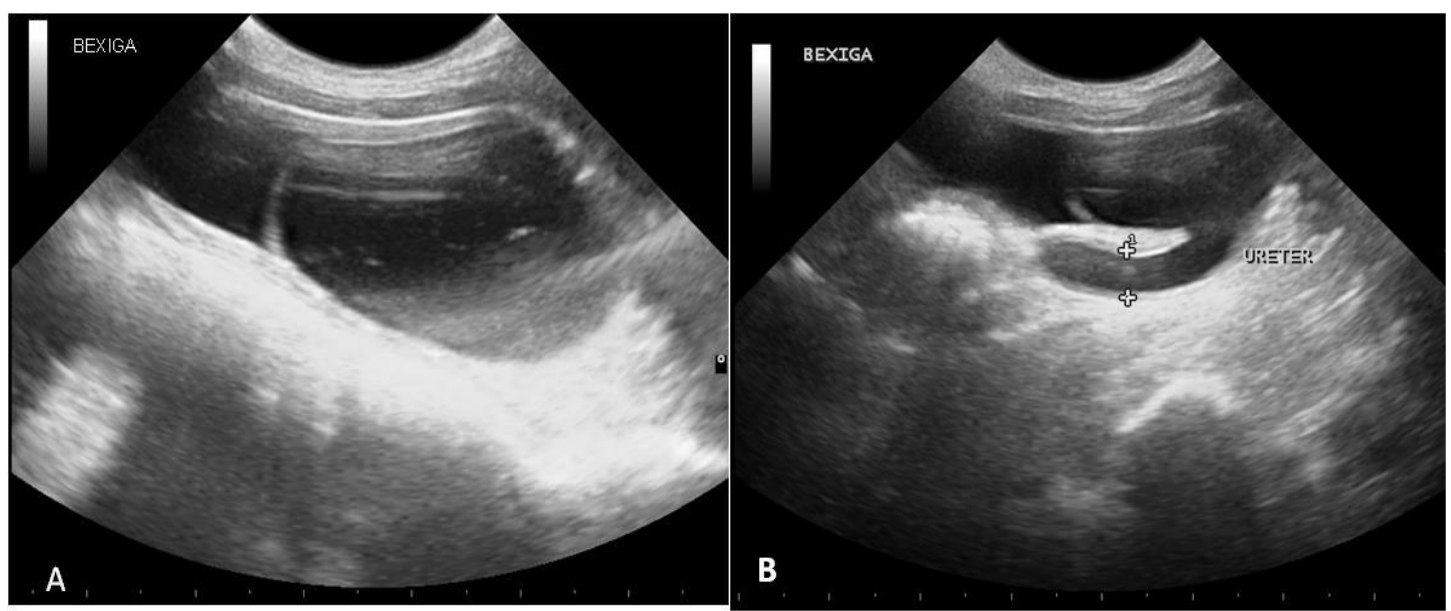

Figura 1. Cão. A- Imagem ultrassonográfica mostra formação cística preenchida por conteúdo anecoico, com parede fina e ecogênica no lúmen vesical (ureterocele). B- Dilatação do ureter esquerdo e sua inserção na região do trígono vesical, comunicando-se com a dilatação cística intravesical.

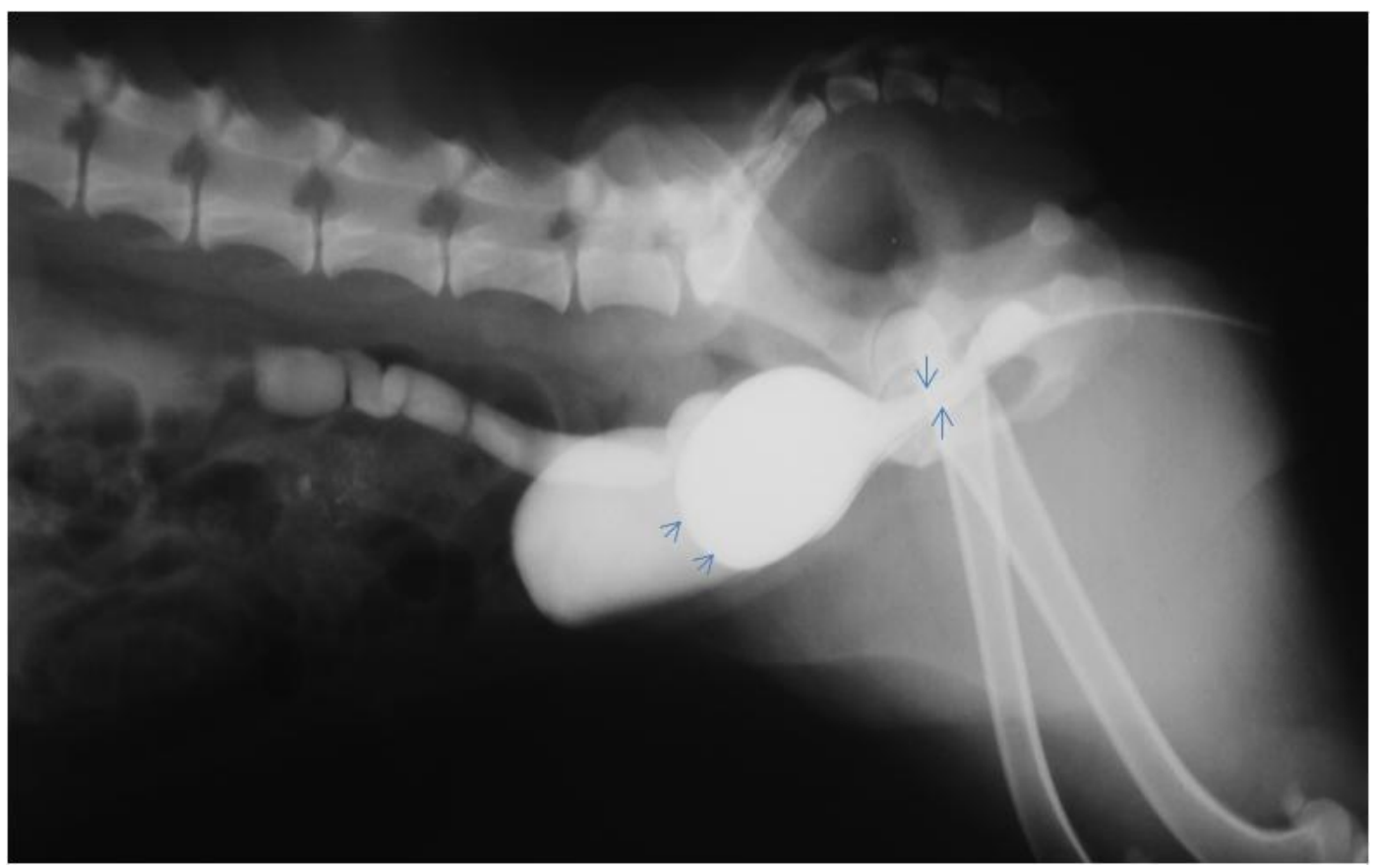

Figura 2. Cão. Cistografia mostra formação cística intravesical preenchida por contraste, prolongando-se para o colo vesical e a uretra (setas). Há refluxo do contraste para o ureter esquerdo dilatado.

Após ser submetido à antibioticoterapia amoxacilina $22 \mathrm{mg} / \mathrm{kg}$, BID/20 dias -, o animal foi encaminhado ao tratamento cirúrgico. Por meio de laparotomia mediana, realizou-se cistotomia ventral e localizou-se o orifício ureteral direito tópico, bem como a dilatação cística do ureter esquerdo com ausência do respectivo orifício ureteral no interior do lúmen vesical, confirmando ureterocele ectópica (Fig. 4A). Somente após a incisão da ureterocele foi possível referendar o orifício ureteral esquerdo (Fig. 4B). Procedeu-se a ureterocelectomia e a sutura da mucosa vesical com fio sintético absorvível (Vicryl ${ }^{\circledR} 4-0$ ), com pontos simples separados, criando-se um novo orifício ureteral esquerdo no lúmen vesical (Fig. 5A). O trajeto ureteral para a uretra foi interrompido por uma ligadura com fio inabsorvível (Prolene ${ }^{\circledR}$ 4-0) (Fig. 5B). 
A cistografia foi realizada de forma habitual e também a ovariossalpingo-histerectomia.

Após o tratamento cirúrgico, houve a resolução do quadro de incontinência urinária, contudo a infecção urinária ainda estava presente. A cultura e o antibiograma indicaram E. coli resistente à amoxacilina, sendo instituído tratamento com enrofloxacina $-5 \mathrm{mg} / \mathrm{kg} / \mathrm{BID} / 15$ dias. A avaliação sonográfica, ocorrida um mês após a ressecção da ureterocele, mostrou que a hidronefrose persistia, embora houvesse diminuído de forma significativa (Fig. 6), e que houve evolução favorável do hidroureter esquerdo, não sendo mais observada a dilatação ureteral. Nessa ocasião, o animal se apresentava em ótimo estado geral.

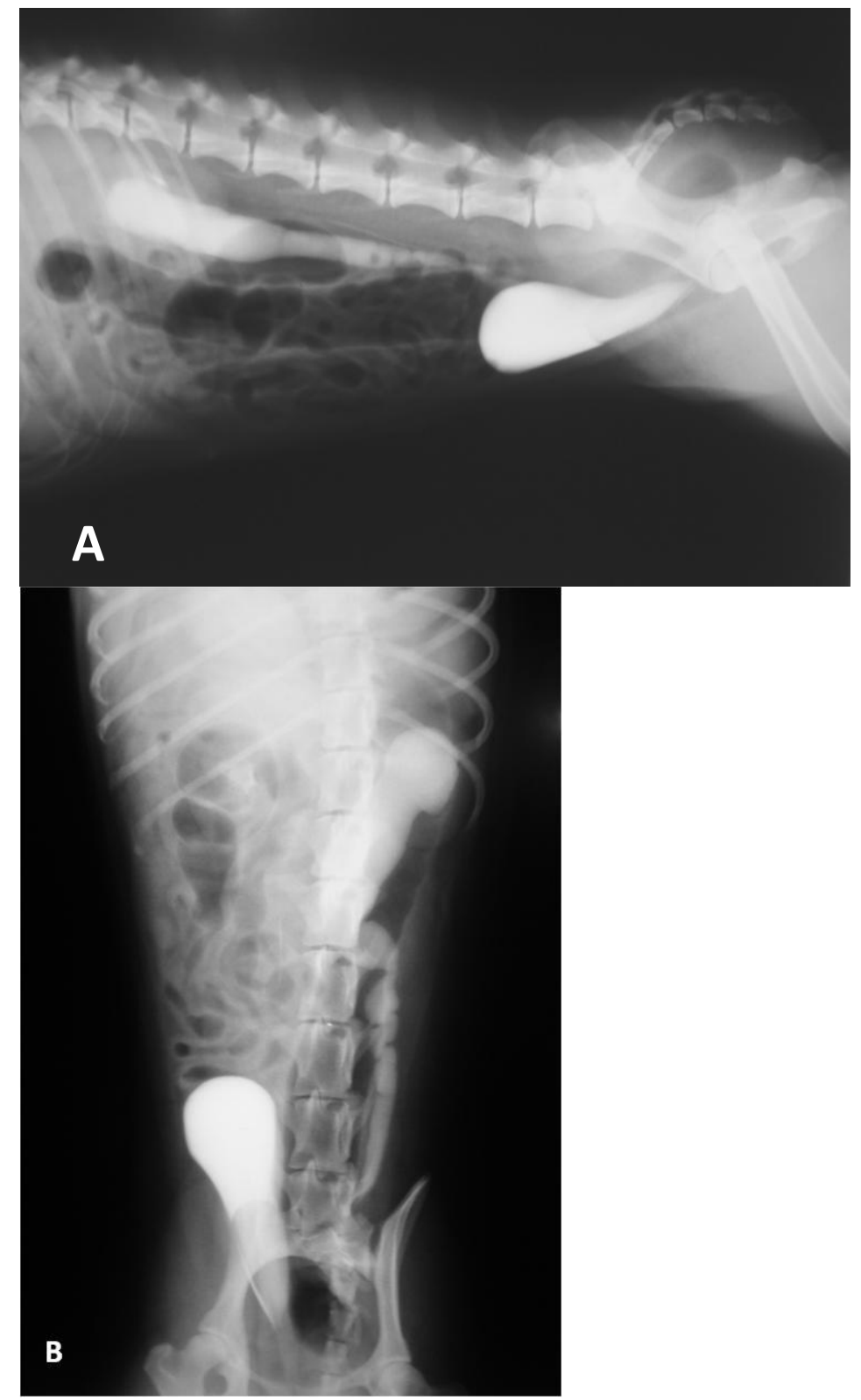

Figura 3. Cão. Urografia excretora 60 minutos após administração do contraste, em projeção laterolateral (A) e ventrodorsal (B). Observa-se acentuada dilatação da pelve e do ureter esquerdos. A ureterocele corresponde à falha de preenchimento de limites bem definidos em região caudal do lúmen vesical. 


\section{Ureterocele ectópica...}
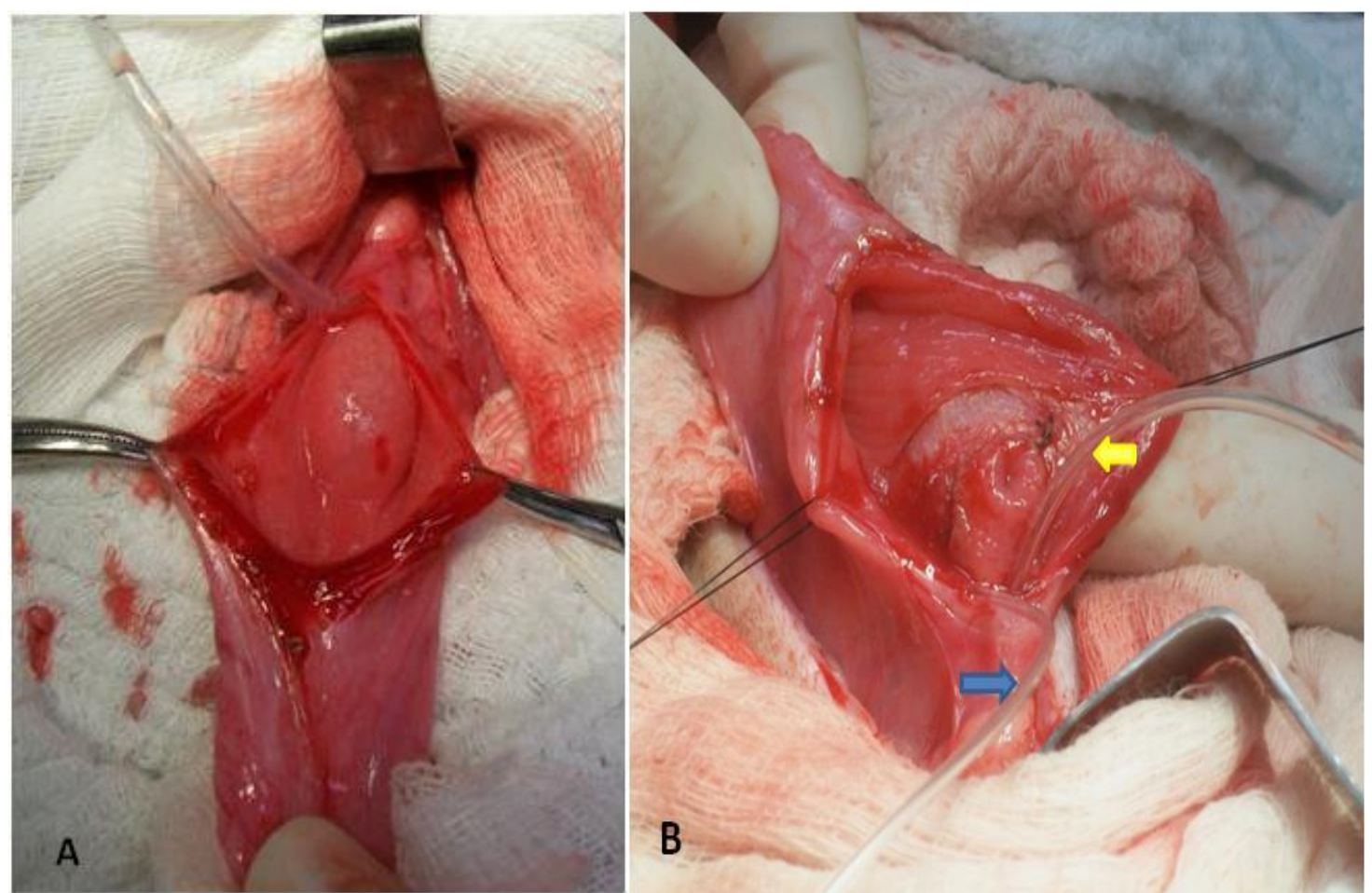

Figura 4. Cão. A- Por uma cistotomia ventral, observa-se a dilatação cística do ureter protruindo-se para o lúmen vesical (ureterocele). B- Abertura da cele para localizar o orifício ureteral esquerdo. As sondas estão referendando o orifício ureteral direito (seta azul) e a uretra (seta amarela).

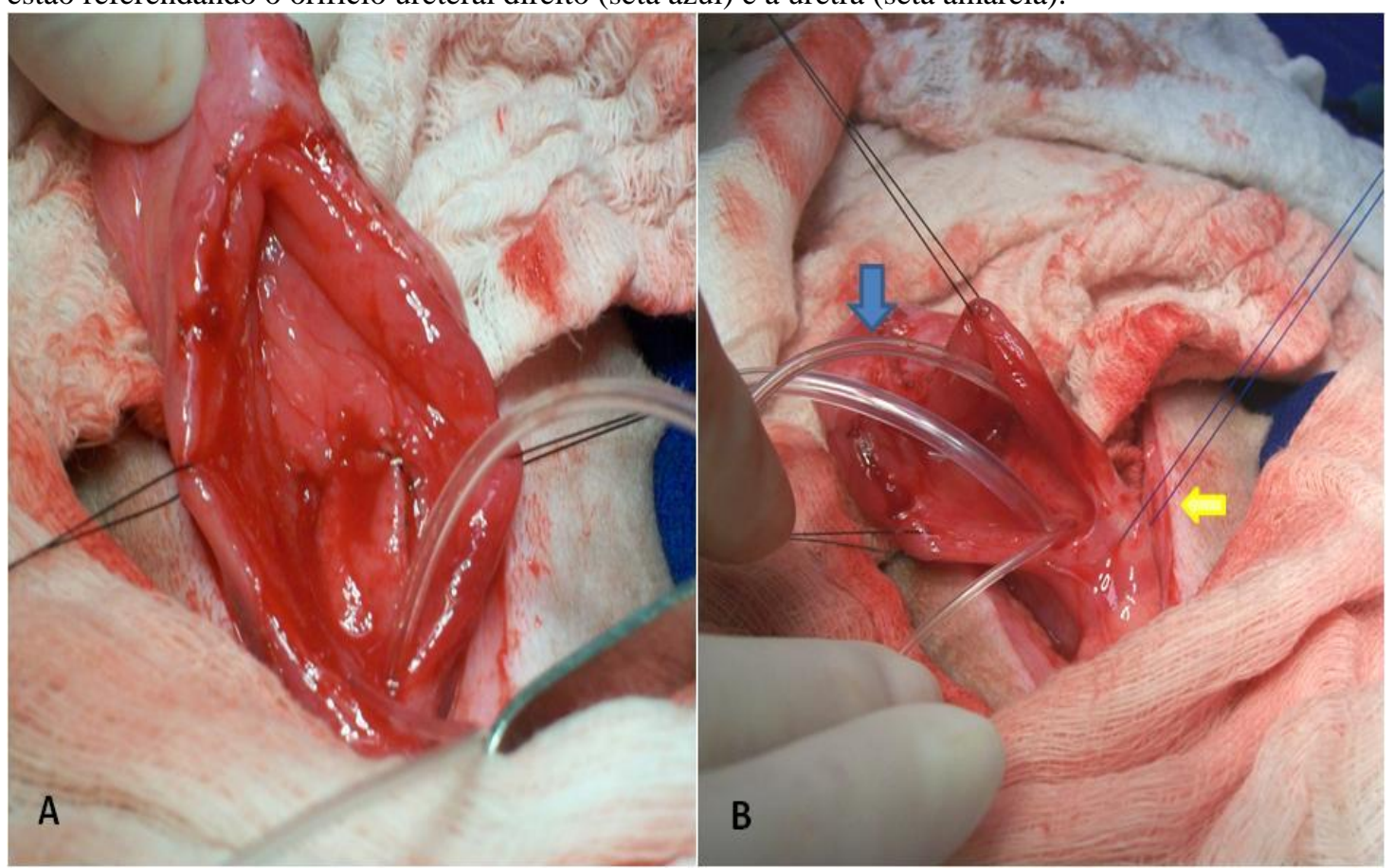

Figura 5. Cão. A- Abertura ureteral esquerda referendada (neoureterostomia) para ressecção da cele. B- A sonda (seta azul) referenda o trajeto ureteral esquerdo para a uretra. Passagem do fio de sutura para ligadura e interrupção desse trajeto (seta amarela). 

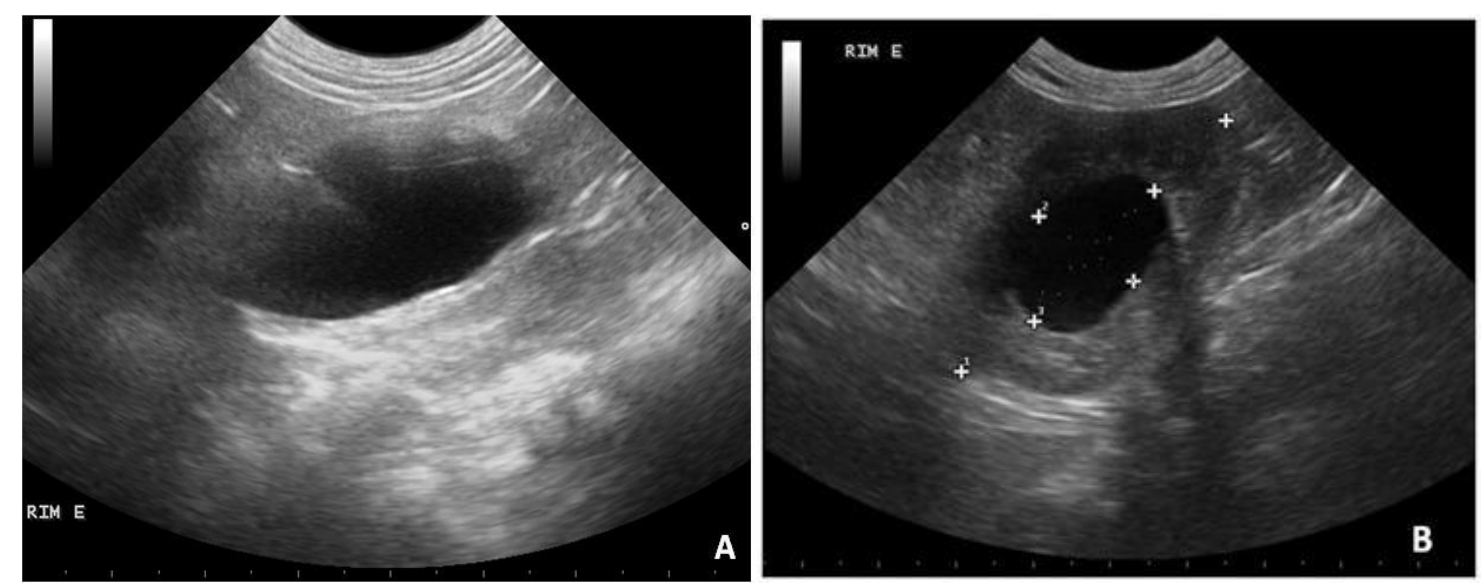

Figura 6. Cão. A- Imagem ultrassonográfica antes do procedimento cirúrgico mostra acentuada dilatação da pelve do rim esquerdo (hidronefrose) com perda da arquitetura renal. B- Ultrassonografia controle do mesmo rim, um mês após o tratamento. Persiste a dilatação da pelve, mas com redução significativa.

\section{DISCUSSÃO}

No desenvolvimento embrionário do sistema urinário dos mamíferos, o ureter se forma a partir do ducto metanéfrico, que deriva da porção distal do mesonefro, o qual, por sua vez, dará origem ao ducto deferente nos machos e se tornará vestigial nas fêmeas. Enquanto a ectopia ureteral pode ser explicada como uma falha na diferenciação dos ductos metanéfrico e mesonéfrico durante a embriogênese (Dyce et al., 2010; Sutherland-Smith et al., 2004a), a causa da ureterocele permanece desconhecida, embora algumas teorias tenham sido propostas (Lautzenhiser e Bjorling, 2002). Alguns autores sugerem que a ureterocele pode estar relacionada com a estenose do orifício ureteral (McLoughlin et al., 1989). Contudo, várias ureteroceles relatadas não cursam com essa alteração, sendo diagnosticados por cistoscopia orifícios ureterais pérvios (Green et al., 2011). Outras explicações, como o retardo da fusão do ducto metanéfrico e as alterações da musculatura da porção terminal do ureter, também têm sido consideradas (McLoughlin et al., 1989).

As ectopias ureterais e as ureteroceles ectópicas podem apresentar o mesmo quadro clínico, na maioria das vezes manifestando-se com incontinência e infecção urinária. A ultrassonografia abdominal foi um exame sensível e específico na detecção da ureterocele e das complicações do trato urinário associadas a ela, como a hidronefrose e o hidroureter (Lamb, 1998; Takiguchi et al., 2007; Secrest et al., 2011), contudo a confirmação de uma ureterocele ectópica não foi possível. A indicação da urografia excretora nesse caso não foi para diagnosticar a ureterocele, já realizada pelo ultrassom, mas sim para avaliar a viabilidade do rim acometido. Além disso, a observação do ureter direito com inserção tópica também foi importante, uma vez que ectopias podem cursar sem dilatações importantes, sendo mais difíceis de serem detectadas pelo exame ultrassonográfico (Sutherland-Smith et al., 2004a). A ureterocele nessa modalidade de exame foi observada como uma falha de preenchimento vesical e, mesmo após uma hora da administração do contraste, não havia sido preenchida de forma satisfatória, portanto também não sendo conclusiva quanto à questão da ectopia. A cistografia não tem sido considerada como um exame de escolha no diagnóstico de ureterocele e foi realizada nesse caso, de certa forma despretensiosamente, por ser um exame de fácil execução, barato e pela oportunidade de ser realizada em um caso relativamente raro. Entretanto, das três modalidades aqui citadas, foi a que melhor sugeriu a presença de uma ureterocele ectópica, sendo possível observar o seu prolongamento em direção ao colo vesical e à uretra. Estudos mais recentes indicam a cistoscopia como o melhor exame na detecção da ectopia ureteral, associada ou não à ureterocele (Green et al., 2011). Infelizmente, a utilização desse exame continua sendo limitada na medicina veterinária.

A técnica cirúrgica requerida no tratamento irá depender das alterações morfofuncionais encontradas em cada caso (Sutherland-Smith et 
al., 2004b; Tattersall e Welsh, 2006). No animal em questão, não houve a necessidade de reimplantação do ureter, uma vez que a junção vesicoureteral estava preservada. As técnicas aqui utilizadas foram a excisão da porção dilatada do ureter (ureterocelectomia) e a criação de um novo orifício ureteral no lúmen vesical (neoureterostomia). A ureteronefrectomia foi descartada, pois o rim esquerdo apresentava filtração, evidenciada pela urografia excretora. Sabe-se que esse é um método qualitativo e grosseiro de se avaliar a filtração glomerular e que bastam $5 \%$ da função renal preservada para se observar radiograficamente a excreção do meio de contraste (Larson, 2009). Contudo, na veterinária, esse exame continua sendo o mais acessível para esse tipo de avaliação, e esta informação foi considerada na conduta do tratamento. Alguns autores questionam a recuperação do peristaltismo de ureteres muito dilatados (Owen, 1973), mas há relatos de resolução ou melhora do grau de hidronefrose e hidroureter após correções cirúrgicas de ureter ectópico (Holt et al., 1982). Embora não se possa avaliar de forma quantitativa a capacidade de recuperação funcional do rim esquerdo após o tratamento, o exame ultrassonográfico demonstrou uma acentuada melhora no aspecto sonográfico do rim e do ureter acometidos.

\section{CONCLUSÕES}

Filhotes que apresentam incontinência urinária devem ser investigados precocemente para se verificar a existência de anormalidades congênitas ureterais. Embora rara, a ureterocele deve ser considerada no diagnóstico diferencial de animais que apresentam esse quadro. Nesse caso, as informações obtidas nas diferentes modalidades diagnósticas por imagem complementam-se e, além do diagnóstico, auxiliam no planejamento do tratamento cirúrgico. O prognóstico dos pacientes é bom quando estes são submetidos ao tratamento cirúrgico. Quanto antes for realizada a cirurgia, maiores são as chances de recuperação da hidronefrose e do hidroureter associados.

\section{REFÊRENCIAS}

ADAMS, L.G.; SYME, H.M. Canine ureteral and lower urinary tract diseases. In: ETTINGER, S.J.; FELDMAN, E. (Eds.) Veterinary Internal Medicine. ST. Louis: Saunders Elsevier, 2010. p.2114.

COLOPY, S.A.; DENNISON, S.E.; LUCCHI, L.J.K.; DANIELSON, K.C. What is your diagnosis? J. Am. Vet. Medical Association, v.238, p.93-294, 2011.

DYCE, K.M.; SACK, W.O.; WENSING, C.J.G. Aparelho Urogenital. In:__. Tratado de Anatomia Veterinária. Rio de Janeiro: Elsevier, 2010. p.164-207.

EISELE, J.G.; JACKSON, J.; HAGER, D. Ectopicureterocele in a cat. J. Am. Animal Hosp. Associat., v.41, p.332-335, 2005.

GREEN, T.A.; ARBLE, J.B.; CHEW, D.J. et al. Diagnosis and management of ureteroceles in two female dogs. J. Am. Anim. Hosp. Associat., v.47, p.138-144, 2011

HOLT, P.E.; GIBBS, C.; PEARSON, H. Canine ectopic ureter- a review of twenty nine cases. $J$. Small Anim. Pract., v.23, p.195-208, 1982, 1982.

JAIMAN, S.; ULHOJ, B.P. Bilateral intravesicalureterocele associated with unilateral partial duplication of the ureter and other anomalies: proposal of a new variant to the classification of ureterocles based on a perinatal autopsy, review of the literature and embryology. J. Compilat., v.118, p.809-814, 2010.

LAMB, C.R. Ultrasonography of the ureters. Vet. Clin. North Am.: Small Anim. Pract., v.28, p.823-847, 1998.

LARSON, M.M. The kidneys and ureters. In: O'Brien R.; Barr, F. Manual of Canine and Feline Abdominal Imaging. BSAVA, 2009. p.185-204.

LAUTZENHISER, S.J.; BJORLING, D.E. Urinary incontinence in a dog with an ectopic ureterocele. J. Am. Anim. Hosp. Associat., v.38, p.29-32, 2002. 
McLOUGHLIN, M.A.; HAUPTMAN. J.G.; SPAULDING, K. Canine Ureterocele: a case report and literature review. J. Am. Anim. Hosp. Associat., v.25, p.699-706, 1989.

OWEN, R. Canine ureteral ectopia - a review. Incidence, diagnosis and treatment. J. Small Anim. Pract., v.14, p.419-427, 1973.

SECREST, S.; BRITT, L.; COOK, C. Imaging diagnosis- bilateral orthotopicureteroceles in a dog. Vet. Radiol. Ultrasound, v.52, p.448-450, 2011.

SILVERMAN, S.; LONG, C.D. The diagnosis of urinary incontinence and abnormal urination in dogs and cats. Vet. Clin. North Am.: Small Anim. Pract., v.30, p.427-447, 2000.

STIFFLER, K.S.; STEVENSON, M.A.; MAHAFFLEY, MB. et al. Intravesicalureterocele with concurrent renal dysfunction in a dog: a case report and proposed classification system. J. Am. Anim. Hosp. Associat.,v.38, p.29-32, 2002.
SUTHERLAND-SMITH, J.; JERRAM, R.M.; WALKER, A.M.; WARMAN, C.G.A. Ectopic ureters and ureteroceles in dogs: presentation, cause and diagnosis. Comp. Cont. Educat. Pract. Vet., v.26, p.303-310, 2004a.

SUTHERLAND-SMITH, J.; JERRAM, R.M.; WALKER, A.M.; WARMAN, C.G.A. Ectopic ureters and ureteroceles in dogs: treatment. Comp. Cont. Educat. Pract. Vet., v.26, p.311-315, 2004 b.

TAKIGUCHI, M.; YASUDA, J.; OCHIAI, K. et al. Ultrasonographic appearance of orthotopic ureterocele in a dog. Vet. Radiol. Ultrasound, v.38, p.398-399, 1997.

TATTERSALL, J.A.; WELSH, E. Ectopic ureterocele in a male dog: a case report and review of surgical management. J. Am. Anim. Hosp. Associat., v.42, p.395-400, 2006. 